\title{
Dorsal Approach for Open Reduction of Complex Metacarpo-phalangeal Joint Dislocations
}

\author{
Pashupati Chaudhary, N Karn, B.P Shrestha, G.P. Khanal, R Maharjan \\ Department of Orthopaedics, B.P.Koirala Institute of Health Sciences, Dharan, Nepal
}

\begin{abstract}
Key words: Complex, Dorsal approach, Metacarpophalangeal Joint

Complex metacarpophalangeal (MP) joint dislocations were classically described by Kaplan ${ }^{1}$ to involve rupture of the volar plate from its weaker proximal attachment to the metacarpal. The volar plate becomes entrapped between the metacarpal head and base of the proximal phalanx by its attachment to the deep transverse metacarpal ligament, thus becoming the primary impediment to reduction. ${ }^{2-6}$ The flexor tendons, pretendinous band of the palmar fascia ulnarly, and lumbrical muscles radially may form a noose around the dislocated MP joint, further inhibiting closed reduction. Initial attempts at reduction using traction will further tighten this envelope, possibly interposing additional structures. This underscores the need for clinical and radiographic recognition of this injury pattern. The radial digital nerve of the index finger is under tension

On examination, the patient with a complex MP joint dislocation will have a relatively benign clinical appearance consisting of mild extension and ulnar deviation at the MP joint, as well as flexion of the interphalangeal (IP) joints (Figure 2). A pathognomonic sign of palmar skin puckering over the head of the metacarpal may be observed. ${ }^{1}$ The posteroanterior (PA) plain radiograph demonstrates dorsal dislocation of the phalangeal base ulnarly ${ }^{5-9}$ (Figure 3A), while the lateral radiograph shows a dorsal dislocation with the MP joint in slight hyperextension (Figure 3B). The presence of sesamoid interposition within the MP joint, best visualized on the oblique radiograph, is pathognomonic. ${ }^{7,-11}$ Open reduction is the treatment of choice for complex MP joint dislocations, as closed as closed reduction is contraindicated
\end{abstract} and often assumes a precarious position between the metacarpal head and the skin, making it susceptible to injury during the volar approach

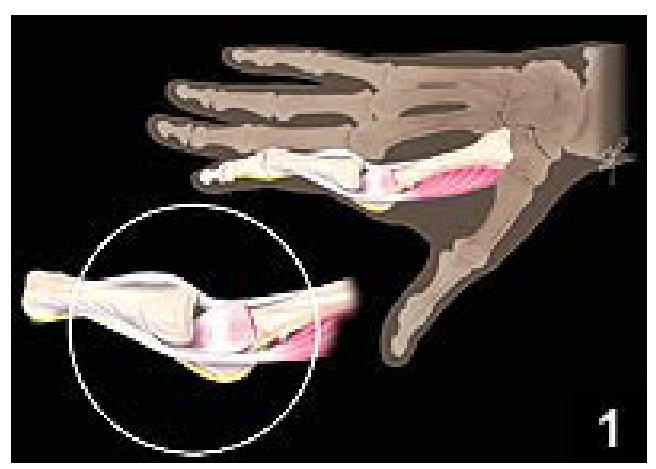

Address for correspondence:

Dr. Pashupati Chaudhary, Assistant Professor Department of Orthopaedics

B.P.Koirala Institute Of Health Sciences, Dharan

PhonePhone-00977-25-525555-3164

E-mail: chaudharypashupati@yahoo.com

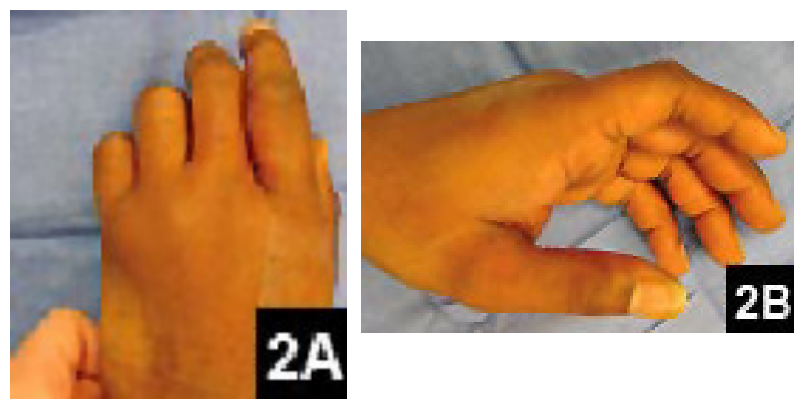

Metacarpophalangeal joints of the fingers are relatively resistant to injury secondary to their strong capsuloligamentous structures and their protected position at the base of the finger. ${ }^{12}$ The joint capsule is supported palmarly by the volar plate, which has a thinner attachment to the metacarpal and a thick fibrocartilaginous attachment to the proximal phalanx. 
Laterally, the MP joint is reinforced by the deep transverse metacarpal and collateral ligaments. ${ }^{13}$ The extrinsic and intrinsic tendons as well as the sagittal bands lend secondary support

Dorsal MP joint dislocations tend to occur most frequently among the exposed border digits, with the index finger most commonly affected, followed by the small finger. ${ }^{14,15}$ The long and ring fingers are protected by the deep transverse metacarpal ligaments and the border digits such that they rarely suffer an isolated dislocation. ${ }^{16}$ Complex MP joint dislocations, by definition, require open reduction. This may be accomplished via either a volar or dorsal approach. Although some articles describe the dorsal approach, ${ }^{3,5,16}$ more frequently cited in the literature is the volar approach. ${ }^{1,2,4,6-8,11,17-24}$ This article reviews the operative technique for open reduction of complex MP joint dislocations using a dorsal

\section{Materials and Methods}

A 25 year old young male, factory worker by occupation from Jhapa district reported to department of Orthopaedics, B.P.Koirala Institute of Health Sciences, Dharan with pain, swelling and deformity of MCP joint of index finger of right hand for 3days. There was alleged history of fall on out-streched hand while working in factory.He took medications for pain from local practitioner but no relief. On clinical examination, there was mild extension and ulnar deviation at the MP joint, as well as flexion of the interphalangeal (IP) joints of right index finger.A pathognomonic sign of palmar skin puckering over the head of the metacarpal was noted. A volar prominence was palpated at the MP joint corresponding to the metacarpal head with a void dorsally. Motor examination of the digit was intact.
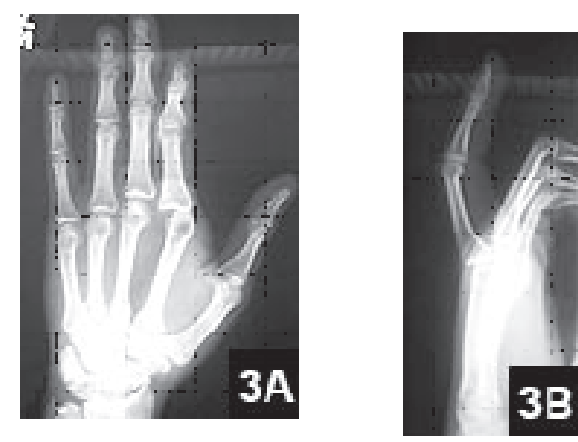

Figure 3: PA (A) and lateral (B) radiographs of the index finger complex MP joint dislocation.
Radiographs demonstrated dorsal dislocation of the proximal phalanx of the index finger without fracture. Multiple attempts at reduction under anesthesia were unsuccessful. Given the clinical and radiographic picture of a complex MP joint dislocation, no further attempts at closed reduction were undertaken. After return to sobriety, we proceeded with operative reduction via a dorsal approach.

\section{Surgical Technique}

An arm tourniquet is applied, and under regional anesthesia the upper extremity is prepped and draped in the usual sterile fashion. A curvilinear incision is made overlying the MP joint (Figure 4). The ulnar sagittal band of the extensor mechanism is incised and later repaired. The capsule is incised longitudinally and inspection of the joint is undertaken (Figure 5). The collateral and accessory collateral ligaments may be imbricated into the joint.

The volar plate is the most common impediment to reduction and must be carefully assessed. Often, the volar plate remains attached to the proximal phalanx and may become completely dorsally translocated over the metacarpal head (Figure 6). Initially the volar plate may be confused with the articular surface of the metacarpal head as it is taut, shiny, and white, with an appearance similar to articular cartilage. Close inspection and proper identification of anatomic structures is critical for proper reduction of the MP joint. Manipulation of the volar plate with a Freer Elevator may be attempted in an effort to reduce the joint maintaining the continuity of the volar plate. More commonly, a longitudinal incision in the volar plate (with articular protection afforded by a Freer Elevator passed over the metacarpal head) will allow it to be reduced over the metacarpal head (Figure 7). The leaflets of the volar plate are allowed to subluxate radial and ulnar to the metacarpal head.
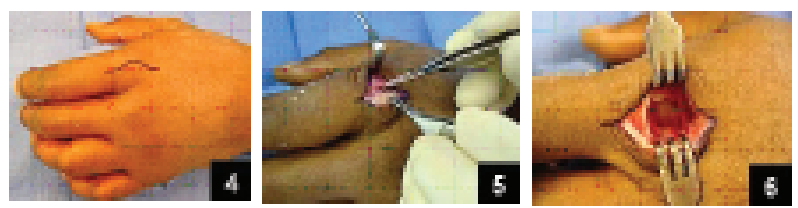

Figure 4: Incision line for dorsal MP joint approach of index finger. Figure 5: Longitudinal capsular incision. Figure 6: Volar plate interposed within MP joint impeding closed reduction. 
As the metacarpal head is being reduced, care must be taken to identify any osteochondral fracture. This allows for a concentric, stable reduction without injury to the articular surfaces. Direct visualization and intraoperative fluoroscopic evaluation confirms a stable reduction through a full arc of motion (Figure 8 ). The capsule is reapproximated with 4-0 Vicryl (Ethicon, Somerville, New Jersey) while the extensor mechanism is reconstituted with 4-0 Tycron (Covidien, Mansfield, Massachusetts) to prevent iatrogenic subluxation. Skin is closed with nonabsorbable horizontal mattress sutures after tourniquet deflation and hemostasis is confirmed.

The patient is then placed into a radial gutter splint with the wrist in gentle extension, the MP joint in $70^{\circ}$ to $90^{\circ}$ of flexion, and the IP joints in extension. Early protected mobilization with a gutter-type splint is initiated after a few days to allow early wound healing. Strengthening begins at 6 weeks to allow for ligamentous healing.
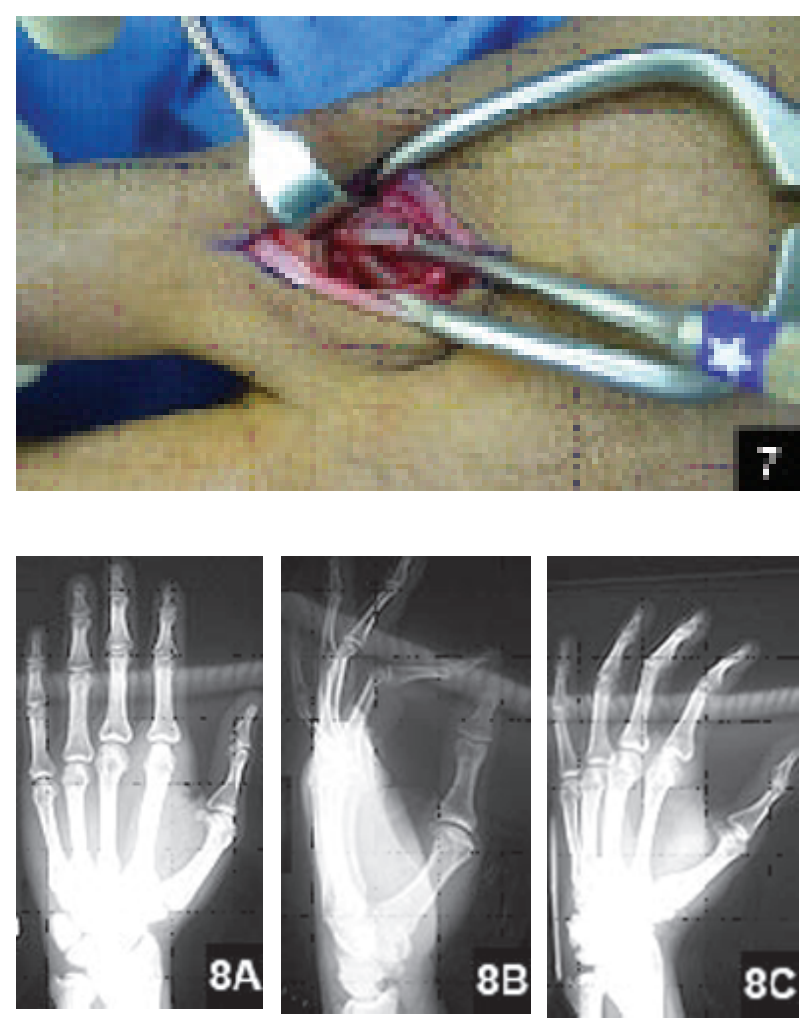

Figure 7: Longitudinal split of volar plate. Figure 8: PA (A), lateral (B), and oblique (C) radiographs, post reduction.

\section{Results}

At 6-week follow-up, the patient's active range of motion consisted of MP joint hyperextension to $5^{\circ}$ and $70^{\circ}$ of flexion, proximal interphalangeal joint extension to $0^{\circ}$ and flexion to $85^{\circ}$, and distal interphalangeal joint extension to $0^{\circ}$ and flexion to $70^{\circ}$. He had $32 \mathrm{lb}$ of grip strength on the left compared to $65 \mathrm{lb}$ on the right. Two-point discrimination was within normal limits. Radiographs demonstrated maintenance of reduction. Despite multiple telephone contacts with the patient, further follow-up was unobtainable; but the patient assured us that he had returned to construction work.

\section{Discussion}

Kaplan $^{1}$ and other authors ${ }^{2,4,6-8,11,17-24}$ described a volar approach to complex MP joint dislocations. This approach requires an extensive release of volar structures, including the volar plate, and places the radial digital nerve at great risk. ${ }^{3,25}$ Other authors ${ }^{3,5,16}$ promoted the dorsal approach due to the increased risk of digital nerve injury in the volar approach. Becton et $\mathrm{al}^{3}$ reported a series of complex MP joint dislocations of the index finger using both volar and dorsal approaches. All 9 patients who underwent a dorsal approach had normal function, while 2 of 3 patients in whom the volar approach was used had anesthesia of the radial aspect of the index finger with limited MP joint range of motion.

The dorsal approach to complex dislocations of the MP joint may offer several advantages over the volar approach. First, there is a reduced risk of injury to the neurovascular bundle, which lies tented volarly between the metacarpal head and skin. ${ }^{4,5,10}$ During the volar approach, damage to the radial neurovascular structure has been well documented in the literature. ${ }^{5,10}$ Furthermore, the management of associated osteochondral fractures, which may be present in up to $50 \%$ of cases, ${ }^{3,5}$ is facilitated by the dorsal approach. Lastly, the dorsal approach allows full visualization of the primary impediment to reduction: the volar plate. It is important to note that the volar plate often drapes dorsally over the metacarpal head, possibly mimicking the articular surface of the metacarpal head.

A potential disadvantage of dorsal open reduction is the need to split the volar plate to reduce the metacarpal head. Theoretically, splitting of the volar plate may delay recovery and reduce stability of the 
healing MP joint. However, there are no published reports of this occurrence. Our rehabilitation protocol ignores this longitudinal split of the volar plate, concentrating on early range of motion.

\section{Conclusion}

The volar and dorsal approaches are viable options in the treatment of complex MP joint dislocations of the fingers. The dorsal approach may offer the critical advantage of decreased risk of neurovascular injury, as well as the ability to manage associated osteochondral fractures. Randomized clinical trials comparing the dorsal and volar approach are needed to further assess their relative effectiveness in treating complex MP joint dislocations.

\section{References}

1. Kaplan EB. Dorsal dislocation of the metacarpophalangeal joint of the index finger. $J$ Bone Joint Surg Am. 1957; 39(5):1081-1086.

2. Johnson AE, Bagg MR. Ipsilateral complex dorsal dislocations of the index and long finger metacarpophalangeal joint. Am J Orthop. 2005; 34(5):241-245.

3. Becton JL, Christian JD Jr, Goodwin HN, Jackson JG III. A simplified technique for treating the complex dislocation of the index metacarpophalangeal joint. J Bone Joint Surg Am. 1975; 57(5):698-700.

4. Barry K, McGee H, Curtin J. Complex dislocation of the metacarpo-phalangeal joint of the index finger: a comparison of the surgical approaches. J Hand Surg Br. 1988; 13(4):466468.

5. Bohart PG, Gelberman RH, Vandell RF, Salamon PB. Complex dislocations of the metacarpophalangeal joint. Clin Orthop Relat Res. 1982; (164):208-210.

6. Adler GA, Light TR. Simultaneous complex dislocation of the metacarpophalangeal joints of the long and index fingers. A case report. $J$ Bone Joint Surg Am. 1981; 63(6):1007-1009.

7. Imbriglia JE, Sciulli R. Open complex metacarpophalangeal joint dislocation. Two cases: index finger and long finger. $J$ Hand Surg Am. 1979; 4(1):72-75.

8. Zemel NP. Metacarpophalangeal joint injuries in fingers. Hand Clin. 1992; 8(4):745-754.

9. Tavin E, Wray RC Jr. Complex dislocation of the index metacarpophalangeal joint with entrapment of a sesamoid. Ann Plast Surg. 1998; 40(1):59-61.

10. Green DP, Terry GC. Complex dislocation of the metacarpophalangeal joint. Correlative pathological anatomy. J Bone Joint Surg Am. 1973; 55(7):1480-1486.

11. Baltas D. Complex dislocation of the metacarpophalangeal joint of the index finger with sesamoid entrapment. Injury. 1995; 26(2):123125.

12. Minami A, An KN, Cooney WP III, Linscheid RL, Chao EY. Ligament stability of the metacarpophalangeal joint: a biomechanical study. J Hand Surg Am. 1985; 10(2):255-260.

13. al-Qattan MM, Robertson GA. An anatomical study of the deep transverse metacarpal ligament. J Anat. 1993; 182(pt 3):443-446.

14. McLaughlin HL. Complex "locked" dislocation of the metacarpophalangeal joints. J Trauma. 1965; 5(6):683-688.

15. Deenstra W. Dorsal dislocation of the metacarpophalangeal joint of the index finger. Neth J Surg. 1981; 33(5):243-246.

16. May JW Jr, Rohrich RJ, Sheppard J. Closed complex dorsal dislocation of the middle finger metacarpophalangeal joint: anatomic considerations and treatment. Plast Reconstr Surg. 1988; 82(4):690-693.

17. al-Qattan MM, Murray KA. An isolated complex dorsal dislocation of the MP joint of the ring finger. J Hand Surg Br. 1994; 19(2):171-173.

18. Hunt JC, Watts HB, Glasgow JD. Dorsal dislocation of the metacarpophalangeal joint of the index finger with particular reference to open dislocation. J Bone Joint Surg Am. 1967; 49(8):1572-1578.

19. Murphy AF, Stark HH. Closed dislocation of the metacarpophalangeal joint of the index finger. 